\title{
A surface Cauchy-Born model for nanoscale materials
}

\author{
Harold S. Park ${ }^{1, *, \dagger}$, Patrick A. Klein ${ }^{2, \ddagger}$ and Gregory J. Wagner ${ }^{2, \S}$ \\ ${ }^{1}$ Department of Civil and Environmental Engineering, Vanderbilt University, Nashville, TN 37235, U.S.A. \\ ${ }^{2}$ Sandia National Laboratories, Livermore, CA 94551, U.S.A.
}

\begin{abstract}
SUMMARY
We present an energy-based continuum model for the analysis of nanoscale materials where surface effects are expected to contribute significantly to the mechanical response. The approach adopts principles utilized in Cauchy-Born constitutive modelling in that the strain energy density of the continuum is derived from an underlying crystal structure and interatomic potential. The key to the success of the proposed method lies in decomposing the potential energy of the material into bulk (volumetric) and surface area components. In doing so, the method naturally satisfies a variational formulation in which the bulk volume and surface area contribute independently to the overall system energy. Because the surface area to volume ratio increases as the length scale of a body decreases, the variational form naturally allows the surface energy to become important at small length scales; this feature allows the accurate representation of size and surface effects on the mechanical response. Finite element simulations utilizing the proposed approach are compared against fully atomistic simulations for verification and validation. Copyright (c) 2006 John Wiley \& Sons, Ltd.
\end{abstract}

KEY WORDS: surface elasticity; Cauchy-Born; surface stress; nanoscale materials

\section{INTRODUCTION}

As nanotechnology has progressed in recent years, the study and creation of nanoscale mechanical structures has become of increasing interest. Many different nanoscale structural elements have been studied and synthesized; examples of these include carbon nanotubes [1], nanowires [2-4], nanoparticles [5,6] and quantum dots [7]. The key manner in which these nanoscale structures are different from those in the bulk (i.e. having a length scale of microns

\footnotetext{
*Correspondence to: Harold S. Park, Department of Civil and Environmental Engineering, Vanderbilt University, Nashville, TN 37235, U.S.A.

${ }^{\dagger}$ E-mail: harold.park@vanderbilt.edu

‡E-mail: patrickklein@mac.com

§E-mail: gjwagne@sandia.gov

Contract/grant sponsor: Publishing Arts Research Council; contract/grant number: 98-1846389

Contract/grant sponsor: United States Department of Energy; contract/grant number: DE-AC04-94AL85000
}

Received 4 January 2006

Revised 10 March 2006

Copyright (c) 2006 John Wiley \& Sons, Ltd.

Accepted 10 March 2006 
or larger) is that the ratio of surface area to volume is dramatically larger for the former case than the latter one.

The unique behaviour of surface-dominated mechanical structures can be seen in many applications. For example, gold nanowires have been seen in molecular dynamics (MD) simulations to undergo phase transformations from an initially face-centred cubic (FCC) $\left\langle\begin{array}{lll}1 & 0 & 0\end{array}\right\rangle$ orientation to body-centred tetragonal (BCT) that are entirely surface stress driven [8,9] once the cross sectional length decreases below about $2 \mathrm{~nm}$. In addition, shape memory $[10,11]$ and pseudoelastic $[10,12,13]$ behaviour has also been recently shown in MD simulations of FCC metal nanowires. Furthermore, the tensile stresses that nanowires can sustain before yielding typically can reach 5-10 GPa $[14,15]$, which are about one order of magnitude larger than bulk materials. Surface effects have also been noticed in quantum dots as errors in the hydrostatic strain calculations of a buried spherical quantum dot can exceed ten percent [16] for dots with sizes around $2 \mathrm{~nm}$. Nanoplates, nanobeams and nanotubes have become structures of interest again due to their dramatic strength increases with decreasing characteristic size; this was illustrated in the seminal experiments of Wong et al. [17].

The difficulty in modelling nanoscale structures is that continuum models, which accurately represent bulk material behaviour, cannot account for the effect of surface stresses without further modification due to the fact that the mechanical behaviour is length scale-independent. Therefore, any attempt at modelling the mechanical behaviour of these nanoscale structures must explicitly incorporate in some manner effects from surface stresses into the mechanical response of the body.

These and other facts motivated previous approaches to modelling surface-dependent elasticity, including those of Gurtin and Murdoch [18], Cammarata et al. [19,20], Miller and Shenoy [21], Shenoy [22], He et al. [23], Sharma et al. [24], Sun and Zhang [25] and Dingreville et al. [26]. Other works are based upon utilization of small atomistic simulations [22,25] to calculate surface stresses on the material. A common thread that connects some of the above works $[21,23,24]$ is that they are based on modifications to the surface elasticity formulation of Gurtin and Murdoch [18], in which a surface stress tensor is introduced to augment the bulk stress tensor typically utilized in continuum mechanics. A complicating factor in this formulation is due to the presence of the surface stress, which creates a coupled system of equations with non-standard boundary conditions. The solution of the coupled field equations combined with the non-classical boundary conditions makes the application of this theory to generalized boundary value problems a challenging task. Furthermore, additional atomistic simulations are generally necessary to produce the elastic constants required for these surface elasticity models.

Similarly, multiple scale modelling of nanoscale materials has had varying degrees of success within the past decade. Methods for both quasistatic [27-29] and dynamic coupling of atomistics and continua [30-40] have been proposed. A crucial issue with these methods is that the continuum region generally surrounds the atomistic region, thereby eliminating the possibility of simulating the effects of surface stresses on the atomistic behaviour. We note that other, strain gradient-type continuum theories exist (see for example References [41-43]) that build a material length scale into the constitutive response. However, those theories aim at capturing size-dependent deformation at the scale of microns due to bulk dislocation motion; the focus of the work here is in capturing size-dependent elasticity at the scale of nanometers where surface stresses act as an effective length scale in controlling the mechanical response.

In this work, we decompose the potential energy of the system into bulk and surface components; while this decomposition has been considered before $[18,19,26]$, those works 
require either higher order terms in the surface energy or empirical fits to constants for the surface stress which require additional atomistic simulations. The uniqueness of the present approach is that the surface energies are obtained directly from an underlying crystal structure and interatomic potential; this approach is adopted since a direct link to the underlying atomic structure is desired for the constitutive response. Therefore, the approach taken in this work uses much of the machinery typically used in Cauchy-Born constitutive modelling [27, 44, 45] with care taken to treat surface unit cells correctly. This modification to treat the surface unit cells differently is the key to utilizing the Cauchy-Born rule to model surface effects in nanostructures as the Cauchy-Born model is based upon a bulk atomic unit cell that observes no free surface effects.

By decomposing the potential energy into bulk and surface components, a variational formulation that is composed of surface and volumetric contributions to the potential energy is obtained. Thus, as the structural length scale decreases and the ratio of surface area to volume increases, the correct surface energy contribution to the overall system energy is naturally obtained. Because the method is based on an energetic approach, the solution of the variational equation can be readily obtained using standard non-linear finite element techniques; as the finite element stresses are simply derivatives of the strain energy, the effects of the surface energies are transferred naturally to the numerical model. This fact constitutes a distinct advantage for the proposed approach as it can therefore be utilized to solve boundary value problems for the deformation of nanoscale materials with arbitrary geometries, surface orientations and external loading. Numerical examples are illustrated in which the proposed continuum model in this work is compared to fully atomistic simulations for verification and validation.

\section{METHODOLOGY}

\subsection{Continuum mechanics preliminaries}

In this section, we briefly review some elements of non-linear continuum mechanics which are central to the Cauchy-Born formulation. The position of a material point $\mathbf{X}$ in the reference configuration can be mapped to the current configuration $\mathbf{x}$ via

$$
\mathbf{x}=\mathbf{X}+\mathbf{u}(\mathbf{X})
$$

where $\mathbf{u}(\mathbf{X})$ is the displacement. The transformation of an infinitesimal line segment from the reference to the current configuration is described by the deformation gradient $\mathbf{F}$, which is defined as

$$
\mathbf{F}=\frac{\partial \mathbf{x}}{\partial \mathbf{X}}=\mathbf{I}+\frac{\partial \mathbf{u}}{\partial \mathbf{X}}
$$

where I is the identity tensor. In Green elastic theory, stress is derived by differentiating the material strain energy density function. In order to satisfy material frame indifference, the strain energy density must be expressed as a function of the right stretch tensor $\mathbf{C}$

$$
W(\mathbf{F})=\Phi(\mathbf{C})
$$

where

$$
\mathbf{C}=\mathbf{F}^{\mathrm{T}} \mathbf{F}
$$


From the strain energy density, one can obtain the first (P) and second (S) Piola-Kirchoff stresses as

$$
\mathbf{P}=\frac{\partial W(\mathbf{F})}{\partial \mathbf{F}} \quad \text { and } \quad \mathbf{S}=2 \frac{\partial \Phi(\mathbf{C})}{\partial \mathbf{C}}
$$

where the Piola-Kirchoff stresses are related by

$$
\mathbf{P}=\mathbf{S F}^{\mathrm{T}}
$$

For crystalline materials, we can construct a strain energy density function by considering the bonds in a representative volume of the crystal. For the case of a centrosymmetric crystal modelled using only pair interactions, the strain energy density is defined in terms of the interatomic potential $U$ as [44]

$$
\Phi(\mathbf{C})=\frac{1}{2} \frac{1}{\Omega_{0}^{a}} \sum_{i=1}^{n_{b}} U\left(r^{(i)}(\mathbf{C})\right)
$$

In (7), $n_{b}$ is the total number of bonds to a representative bulk atom, $\Omega_{0}^{a}$ is the representative atomic volume in the undeformed configuration, $r^{(i)}$ is the deformed bond length which follows the relationship

$$
r^{(i)}=\sqrt{\mathbf{R}_{0}^{(i)} \cdot \mathbf{C} \mathbf{R}_{0}^{(i)}}
$$

where $\mathbf{R}_{0}$ is the undeformed bond vector, and the factor of $1 / 2$ in (7) comes from splitting the energy of each bond. More discussion on this factor of $1 / 2$ will be made in the next section.

The strain energy density (7) is exact in describing the change in energy per volume of a bulk atom in a corresponding defect-free atomistic system subject to homogeneous deformation. The approach can also be applied to more complicated interatomic interactions such as embedded atom (EAM) potentials [46], silicon [47], or carbon nanotubes [45, 48]. From (5) and (7), the second Piola-Kirchoff stress is given by

$$
\mathbf{S}(\mathbf{C})=\frac{1}{\Omega_{0}^{a}} \sum_{i=1}^{n_{b}}\left(U^{\prime}\left(r^{(i)}\right) \frac{\partial r^{(i)}}{\partial \mathbf{C}}\right)
$$

These assumptions constitute the Cauchy-Born hypothesis.

As mentioned above, all points at which the Cauchy-Born hypothesis is applied are assumed to lie in the bulk because $\Phi(\mathbf{C})$ does not account for surface effects. Therefore, the issue at hand is to develop an expression for the energy density along the surfaces of a body. If the effects of surfaces are not considered, a standard bulk Cauchy-Born relationship can be utilized to derive the stress [27] as described above in (9). Once the surface and bulk energy densities are determined, the stresses arising from bulk and surface energetics can be defined using the relationships (5) and (9) defined in this section.

\subsection{Surface and bulk energy densities}

In this section, we discuss the methodology by which the total atomistic potential energy of a body is represented by continuum energy densities with appropriate representations for bulk and surface energy densities. The relationship between the continuum strain energy and the 


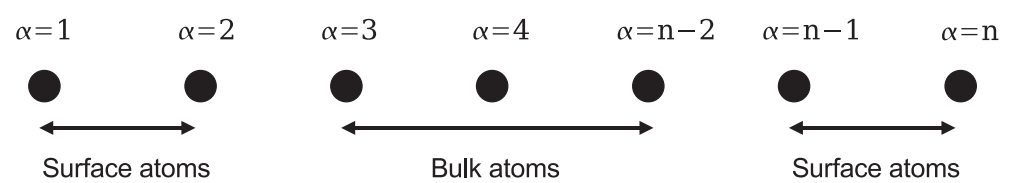

Figure 1. Illustration of bulk and surface atoms for a 1D atomic chain with second nearest neighbour interactions.

total potential energy of the corresponding, defect-free atomistic system can be approximated as

$$
\sum_{\alpha=1}^{n_{\text {atoms }}} U_{\alpha}(r) \approx \int_{\Omega_{0}^{\text {bulk }}} \Phi(\mathbf{C}) \mathrm{d} \Omega+\int_{\Gamma_{0}} \gamma(\mathbf{C}) \mathrm{d} \Gamma
$$

where $U_{\alpha}$ is the potential energy of atom $\alpha, r$ is the interatomic distance, $\Phi(\mathbf{C})$ is the bulk strain energy density introduced in Section 2.1, $\Omega_{0}^{\text {bulk }}$ represents the volume of the body in which all atoms are fully co-ordinated, $\gamma(\mathbf{C})$ is the surface energy density, $\Gamma_{0}$ represents the surface area of the body in which the atoms are underco-ordinated and $n_{\text {atoms }}$ is the total number of atoms in the system.

Analogous to the bulk energy density, we will derive this surface energy density $\gamma(\mathbf{C})$ to describe the energy per representative undeformed area of atoms at or near the surface of a homogeneously deforming crystal. Figure 1 illustrates the decomposition given by (10). The bulk strain energy density function $\Phi(\mathbf{C})$ is integrated only over the part of the domain composed of fully co-ordinated atoms, or atoms $\alpha=3 \rightarrow n-2$ in Figure 1. The potential energy of the atoms at or near the surface (atoms $\alpha=1,2, n-1, n$ in Figure 1) which do not possess a bulk bonding configuration is represented by the surface energy density $\gamma(\mathbf{C})$. In order to derive the surface energy density $\gamma(\mathbf{C})$ with a Cauchy-Born approach, we need to identify the surface unit cell, or the cluster of atoms that reproduce the structure of the surface layers when repeated in the plane of the surface. The surface unit cell possesses translational symmetry only in the plane of the surface, unlike the bulk unit cell which possesses translational symmetry in all directions. As illustrated in the Figure 1, each layer of atoms near the surface has a different bonding configuration. With these considerations, we express the surface energy density generally as

$$
\gamma(\mathbf{C})=\frac{1}{2} \frac{1}{\Gamma_{0}^{a}} \sum_{i=1}^{n_{\mathrm{sl}}} \sum_{j=1}^{n_{b_{i}}} U\left(r^{(j)}(\mathbf{C})\right)
$$

where $n_{\mathrm{sl}}$ is the number of surface layers, $n_{b_{i}}$ is the number of bonds for atoms in surface layer $i, \Gamma_{0}^{a}$ is the representative area of the entire surface layer cluster and the factor of $1 / 2$ again comes due to splitting the energy of each bond. In Sections 2.3 and 2.4, we will convert the discrete summation in (11) into a continuum approximation of surface energy density while explicitly decomposing the energy density into an appropriate expression for different surface layers.

The surface energy density as written in (11) is valid for FCC lattices, or lattices which have one atom per surface unit cell. For alloyed systems or lattices such as graphene which will require more than one atom per surface unit cell, additional kinematic, or internal degrees of freedom must be introduced. These modifications have been made in previous Cauchy-Born 
extensions $[47,48]$, and can be similarly incorporated into the present work. However, we will concentrate here on those lattices which contain one atom per surface unit cell.

We note that in Equations (7) and (11), which describe the energies for bulk and surface configurations, respectively, we have split all the interaction energies by a factor of $1 / 2$. The reason this is done is such that the energies can be treated as in a fully atomistic calculation, i.e. atoms which lie on or near the surface will interact with all their neighbours, including atoms in the bulk and other surface atoms, and vice versa; no special rules for interactions need be introduced for either the bulk or surface energy densities. By doing this, the issue of double counting bond energies will naturally be avoided just as it would be in an atomistic simulation.

We can immediately define the surface stress resulting from the surface energy in (11) as

$$
\tilde{\mathbf{S}}(\mathbf{C})=2 \frac{\partial \gamma(\mathbf{C})}{\partial \mathbf{C}}=\frac{1}{\Gamma_{0}^{a}} \sum_{i=1}^{n_{\mathrm{s} 1}} \sum_{j=1}^{n_{b_{i}}}\left(U^{\prime}\left(r^{(j)}\right) \frac{\partial r^{(j)}}{\partial \mathbf{C}}\right)
$$

We call this stress $\tilde{\mathbf{S}}(\mathbf{C})$ a surface stress because it is not a stress in the traditional sense as the normalization factor is an area, instead of a volume. In addition, $\tilde{\mathbf{S}}(\mathbf{C})$ is a $3 \times 3$ tensor with normal components, which differs from the $2 \times 2$ surface stress tensor with tangential components traditionally used in surface elasticity [22,24]; the normal component of $\tilde{\mathbf{S}}(\mathbf{C})$ arises naturally due to underco-ordinated atoms lying at a material surface, and causes surface relaxation as occurs in nanoscale materials. While the significance of the surface stress will be discussed in detail later, the key idea is that because it is derived directly from the surface energy, as the surface energy portion of the total energy increases, as occurs in nanoscale systems, the surface stress will also become significantly relative to the bulk stress.

Analogous to the Cauchy-Born approach applied to the bulk, we assume the configuration of the deformed surface cluster can be determined by mapping the cluster to the deformed configuration with the deformation gradient $\mathbf{F}$. Unlike the bulk case, the lack of translational symmetry in the direction normal to the surface implies the layers will deform non-uniformly in this direction for a given homogeneous deformation in the plane of the surface. This nonuniform relaxation could be represented in $\gamma(\mathbf{C})$ by introducing internal degrees of freedom, analogous to how internal degrees of freedom have been used when applying the Cauchy-Born approach to silicon [47] or carbon nanotubes [48]. However, the focus of this study is to validate the basic approach of applying the Cauchy-Born hypothesis to surface layers. The introduction of internal degrees of freedom to improve the accuracy of the surface energy density function will therefore be the subject of future study. We also note that the surface clusters only apply away from edges, or lines where surfaces intersect. One could also identify vertex atoms lying where edges intersect. The representative cluster approach taken for surfaces could be extended to edges and vertices as well, though these are not explored here.

In the next two sections, we illustrate how to calculate the surface energy density $\gamma(\mathbf{C})$ in both one- and three-dimensions, beginning with the one-dimensional case for clarity.

\section{3. $1 D$ formulation}

In this section, we illustrate the approach in 1D for simplicity and clarity. First define a 1D chain of atoms going from $\alpha=1 \rightarrow n$ with $n$ being the total number of atoms in the 1D chain as in Figure 1. We assume that the atoms interact with their nearest and second nearest 
neighbours. Because of the second nearest interactions, we can decompose the total energy density of this 1D nanostructure as a sum of bulk and surface components following (10) as

$$
\begin{aligned}
\int_{\Omega_{0}^{\text {bulk }}} \Phi(C) \mathrm{d} \Omega & =\sum_{\alpha=3}^{n-2} \Phi_{\alpha}(C) \\
\int_{\Gamma_{0}} \gamma(C) \mathrm{d} \Gamma & =\int_{\Gamma_{0}^{1}} \gamma_{\Gamma_{0}^{1}}(C) \mathrm{d} \Gamma+\int_{\Gamma_{0}^{2}} \gamma_{\Gamma_{0}^{2}}(C) \mathrm{d} \Gamma
\end{aligned}
$$

The surface energy densities $\gamma_{\Gamma_{0}^{1}}(C)$ and $\gamma_{\Gamma_{0}^{2}}(C)$ contain contributions from all atoms that are not in a fully-co-ordinated bulk configuration. Because the energies for the atoms lying on the material surface will differ from those atoms lying one lattice parameter into the bulk, we can explicitly write the surface energy densities as

$$
\begin{aligned}
& \gamma_{\Gamma_{0}^{1}}(C)=\gamma_{1}(C)+\gamma_{n}(C) \\
& \gamma_{\Gamma_{0}^{2}}(C)=\gamma_{2}(C)+\gamma_{n-1}(C)
\end{aligned}
$$

where

$$
\begin{aligned}
& \gamma_{1}(C)=\gamma_{n}(C)=\frac{1}{2} \frac{1}{\Gamma_{0}^{a^{1}}} \sum_{i=1}^{2} U\left(r^{(i)}(C)\right) \\
& \gamma_{2}(C)=\gamma_{n-1}(C)=\frac{1}{2} \frac{1}{\Gamma_{0}^{a^{2}}} \sum_{i=1}^{3} U\left(r^{(i)}(C)\right) \\
& \Phi_{\alpha}(C)=\frac{1}{2} \frac{1}{\Omega_{0}^{a}} \sum_{i=1}^{4} U\left(r^{(i)}(C)\right)
\end{aligned}
$$

and the factor of $1 / 2$ comes from splitting the energy of each bond; (17) and (18) demonstrate that the surface energy densities may vary depending on the number of neighbours and the reference area occupied $\left(\left(\Gamma_{0}^{a^{1}}\right)\right.$ and $\left.\left(\Gamma_{0}^{a^{2}}\right)\right)$ for each surface unit cell where in 1D, the reference areas $\Gamma_{0}^{a^{1}}$ and $\Gamma_{0}^{a^{2}}$ are unity. Note that bulk atoms have four neighbours, while atoms on the surface have two and atoms one lattice parameter into the bulk have three for second nearest neighbour interactions.

Having defined the energy densities for bulk and surface components, we can integrate the energy densities in (17)-(19) to modify (10) and obtain the Cauchy-Born energy equivalence including surface effects

$$
\sum_{\alpha=1}^{n_{\text {atoms }}} U_{\alpha}(r)=\int_{\Omega_{0}^{\text {bulk }}} \Phi(C) \mathrm{d} \Omega+\int_{\Gamma_{0}^{1}} \gamma_{\Gamma_{0}^{1}}(C) \mathrm{d} \Gamma+\int_{\Gamma_{0}^{2}} \gamma_{\Gamma_{0}^{2}}(C) \mathrm{d} \Gamma
$$

In (20), it is understood that $\Omega_{0}^{\text {bulk }}$ represents the volume occupied by atoms $\alpha=3 \rightarrow n-2$, while the area integrals $\Gamma_{0}^{1}$ and $\Gamma_{0}^{2}$ represent the area occupied by atoms $\alpha=1, n$ and $\alpha=2, n-1$, respectively. Note that the discrete representation of the surface energy in (11) has been modified into two continuous terms in (20) to account for the fact that atoms in surface layers $\Gamma_{0}^{1}$ and $\Gamma_{0}^{2}$ will require different expressions for the surface energy density. 


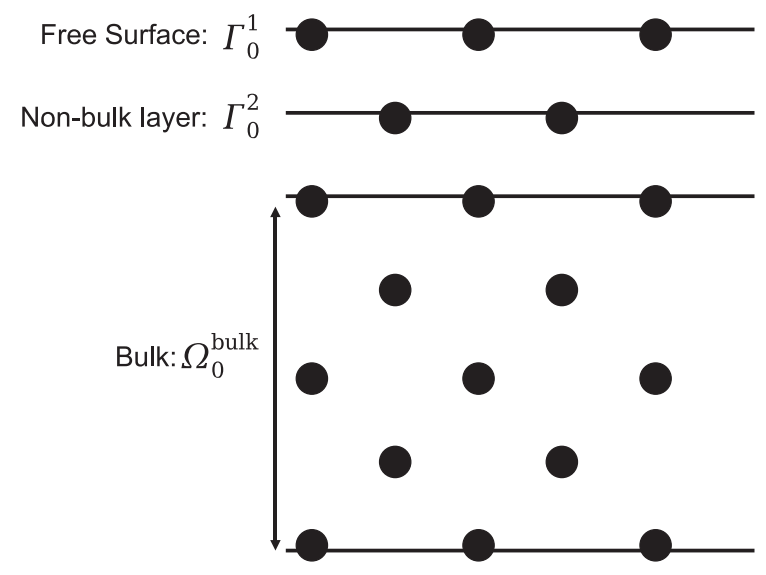

Figure 2. Illustration of bulk and non-bulk layers of atoms in a 3D FCC crystal with fourth shell interactions.

By considering external work on the system, the total potential energy $\Pi(u)$ of the system is written as

$$
\Pi(u)=\int_{\Omega_{0}^{\text {bulk }}} \Phi(C) \mathrm{d} \Omega+\int_{\Gamma_{0}^{1}} \gamma_{\Gamma_{0}^{1}}(C) \mathrm{d} \Gamma+\int_{\Gamma_{0}^{2}} \gamma_{\Gamma_{0}^{2}}(C) \mathrm{d} \Gamma-\int_{\Gamma_{0}} T u \mathrm{~d} \Gamma
$$

where $T$ is the external traction acting on the traction boundary $\Gamma_{0}$. A critical point to note is that while the total potential energy of the system as written in (21) contains two surface integrals, the energies of those surface layers include all interactions with neighbouring bulk and surface atoms as can be seen in (17) and (18). In three dimensions, the surface layer energies also account for out of plane interactions as would occur for a fully atomistic system, and are discussed next.

\section{4. $3 D$ formulation}

In 3D, we assume that the atomistic interactions extend out to fourth shell interactions; the radius of the $n$th shell can be written as $d_{n}=a_{0} \sqrt{n / 2}$ where $a_{0}$ is the lattice parameter. The first shell contains 12 atoms, the second shell contains 6 atoms, the third shell contains 24 atoms and the fourth shell contains 12 atoms. The energetic decomposition is illustrated in Figure 2 for a cross section of a $\langle 100\rangle$ oriented FCC crystal showing a $\{100\}$ face. Because of the fourth shell interactions, the atoms in layers $\Gamma_{0}^{1}$ and $\Gamma_{0}^{2}$ are not fully co-ordinated, i.e. do not have the same number of atomic neighbours as those lying in the bulk. Atoms lying in the third layer and greater (within $\Omega_{0}^{\text {bulk }}$ in Figure 2) are at a bulk bonding configuration as they feel a full complement of atomic neighbours.

The idea then is that an equivalent continuum representation of the energy can be constructed from three distinct entities. The first corresponds to the representation of the bulk atoms within $\Omega_{0}^{\text {bulk }}$ in Figure 2; because all the atoms in the bulk have the same energy in the undeformed configuration, the bulk energy density can be calculated by summing over all neighbouring 
atoms and normalizing by the initial volume $\Omega_{0}^{a}$ of a reference unit cell

$$
\Phi(\mathbf{C})=\frac{1}{2} \frac{1}{\Omega_{0}^{a}} \sum_{i=1}^{n_{\text {bulk }}} U\left(r^{(i)}(\mathbf{C})\right)
$$

where $\Phi(\mathbf{C})$ represents the bulk energy density and $n_{\text {bulk }}$ is the number of neighbours for an atom lying in the bulk; $n_{\text {bulk }}=54$ for fourth shell interactions within an FCC crystal.

The second and third distinct entities are the atoms in layers $\Gamma_{0}^{1}$ and $\Gamma_{0}^{2}$ that do not have a full complement of atomic neighbours. Because of this, care must be taken to modify the sum over neighbours to reflect the non-bulk configuration. In addition, we normalize the energies for these surface atoms not by the reference volume, but instead by a reference area. This area normalization is key to this approach; by allowing the surface energies to depend on the area, we will be able to capture length scale dependent surface effects as the surface energies will contribute significantly to the total energy as the size of structures approaches the nanoscale. The surface energy densities for layers $\Gamma_{0}^{1}$ and $\Gamma_{0}^{2}$ can thus be written as

$$
\int_{\Gamma_{0}} \gamma(\mathbf{C}) \mathrm{d} \Gamma=\int_{\Gamma_{0}^{1}} \gamma_{\Gamma_{0}^{1}}(\mathbf{C}) \mathrm{d} \Gamma+\int_{\Gamma_{0}^{2}} \gamma_{\Gamma_{0}^{2}}(\mathbf{C}) \mathrm{d} \Gamma
$$

where

$$
\begin{aligned}
& \gamma_{\Gamma_{0}^{1}}(\mathbf{C})=\frac{1}{2} \frac{1}{\Gamma_{0}^{a^{1}}} \sum_{i=1}^{n} U_{\Gamma_{0}^{1}}\left(r^{(i)}(\mathbf{C})\right) \\
& \gamma_{\Gamma_{0}^{2}}(\mathbf{C})=\frac{1}{2} \frac{1}{\Gamma_{0}^{a^{2}}} \sum_{i=1}^{n} U_{\Gamma_{0}^{2}}\left(r^{(i)}(\mathbf{C})\right)
\end{aligned}
$$

Like the one-dimensional case, the surface energy densities vary depending on the number of neighbours, where $n_{\Gamma_{0}^{1}}=33$ and $n_{\Gamma_{0}^{2}}=45$ for fourth shell interactions within a $\langle 100\rangle$ oriented FCC crystal with $\left\{\begin{array}{lll}1 & 0 & 0\end{array}\right\}$ side surfaces, and also the reference areas $\Gamma_{0}^{a^{1}}$ and $\Gamma_{0}^{a^{2}}$ occupied by each surface unit cell. We note again that while the surface energy densities (24) and (25) are normalized by area, the energies contain contributions from all neighbouring atoms, including out of plane contributions from other surface and bulk unit cells as would be standard for a fully atomistic system.

We note that the expressions (24) and (25) for the surface energy densities are completely general for lattices that contain only one atom per surface unit cell, and do not impose any restriction on the surface orientations that can be modelled for those lattices. In particular, for side surfaces with different orientations, the surface unit cell simply needs to be adjusted to match the crystal structure for the given surface orientation. Specifically, in (24) and (25), the energy function $U$ will remain the same, while the summations over the number of neighbours $n_{\Gamma_{0}^{1}}$ and $n_{\Gamma_{0}^{2}}$ may vary depending on the surface orientation. This point is critical, and implies that the proposed method can be utilized to study the effects of different surface orientations and crystal structures on the mechanical strength and properties of nanostructured materials; this will be investigated in future publications. 
Having defined the energy densities for the bulk and surface components, we can further integrate the energy densities in (22), (24) and (25) to modify (10) and obtain the Cauchy-Born energy equivalence including surface effects

$$
\sum_{\alpha=1}^{n_{\text {atoms }}} U_{\alpha}(r)=\int_{\Omega_{0}^{\text {bulk }}} \Phi(\mathbf{C}) \mathrm{d} \Omega+\int_{\Gamma_{0}^{1}} \gamma_{\Gamma_{0}^{1}}(\mathbf{C}) \mathrm{d} \Gamma+\int_{\Gamma_{0}^{2}} \gamma_{\Gamma_{0}^{2}}(\mathbf{C}) \mathrm{d} \Gamma
$$

We now consider the effects of external work on the system in order to write the complete potential energy $\Pi(\mathbf{u})$ of the system

$$
\Pi(\mathbf{u})=\int_{\Omega_{0}^{\text {bulk }}} \Phi(\mathbf{C}) \mathrm{d} \Omega+\int_{\Gamma_{0}^{1}} \gamma_{\Gamma_{0}^{1}}(\mathbf{C}) \mathrm{d} \Gamma+\int_{\Gamma_{0}^{2}} \gamma_{\Gamma_{0}^{2}}(\mathbf{C}) \mathrm{d} \Gamma-\int_{\Gamma_{0}}(\mathbf{T} \cdot \mathbf{u}) \mathrm{d} \Gamma
$$

where $\mathbf{T}$ is the external traction acting upon the traction boundary $\Gamma_{0}$.

\section{FINITE ELEMENT FORMULATION AND IMPLEMENTATION}

\subsection{Variational formulation}

In this section, we derive the variational formulation from which the finite element (FE) equilibrium equations can be obtained. We begin with the total potential energy of the system as written in (27)

$$
\Pi(\mathbf{u})=\int_{\Omega_{0}^{\text {bulk }}} \Phi(\mathbf{C}) \mathrm{d} \Omega+\int_{\Gamma_{0}^{1}} \gamma_{\Gamma_{0}^{1}}(\mathbf{C}) \mathrm{d} \Gamma+\int_{\Gamma_{0}^{2}} \gamma_{\Gamma_{0}^{2}}(\mathbf{C}) \mathrm{d} \Gamma-\int_{\Gamma_{0}}(\mathbf{T} \cdot \mathbf{u}) \mathrm{d} \Gamma
$$

In order to obtain a form suitable for FE calculations, we introduce the standard discretization of the displacement field $\mathbf{u}(\mathbf{X})$ using FE shape functions as

$$
\mathbf{u}(\mathbf{X})=\sum_{I=1}^{n n} N_{I}(\mathbf{X}) \mathbf{u}_{I}
$$

where $N_{I}$ are the shape or interpolation functions, $n n$ are the total number of nodes in the discretized continuum, and $\mathbf{u}_{I}$ are the displacements of node $I[49,50]$. Substituting (4) and (29) into (28) and differentiating gives the minimizer of the potential energy and also the FE nodal force balance [49]

$$
\frac{\partial \Pi}{\partial \mathbf{u}_{I}}=\int_{\Omega_{0}^{\text {bulk }}} \mathbf{B}^{\mathrm{T}} \mathbf{S} \mathbf{F}^{\mathrm{T}} \mathrm{d} \Omega+\int_{\Gamma_{0}^{1}} \mathbf{B}^{\mathrm{T}} \tilde{\mathbf{S}}^{(1)} \mathbf{F}^{\mathrm{T}} \mathrm{d} \Gamma+\int_{\Gamma_{0}^{2}} \mathbf{B}^{\mathrm{T}} \tilde{\mathbf{S}}^{(2)} \mathbf{F}^{\mathrm{T}} \mathrm{d} \Gamma-\int_{\Gamma_{0}} N_{I} \mathbf{T} \mathrm{d} \Gamma
$$

where $\mathbf{S}$ is the second Piola-Kirchoff stress due to the bulk strain energy, $\mathbf{B}^{\mathrm{T}}=\left(\partial N_{I} / \partial \mathbf{X}\right)^{\mathrm{T}}$ and $\tilde{\mathbf{S}}^{(i)}$ are surface stresses, similar to (12) and of the form

$$
\tilde{\mathbf{S}}^{(i)}(\mathbf{C})=\frac{1}{\Gamma_{0}^{a^{i}}} \sum_{j=1}^{n_{b_{i}}}\left(U^{\prime}\left(r^{(j)}\right) \frac{\partial r^{(j)}}{\partial \mathbf{C}}\right)
$$

In (31), $\Gamma_{0}^{a^{i}}$ represents the normalizing area for atomic layer $i$, and $n_{b_{i}}$ represents the number of bonds for an atom lying within atomic layer $i$, which may differ depending on whether the 
atom lies on the first or second layer of surface atoms as discussed in Section 2.4. We call these stresses surface stresses because they are not stresses in the traditional sense, i.e. the normalization factor is an area, instead of a volume. In addition, the surface stresses $\tilde{\mathbf{S}}^{(i)}(\mathbf{C})$ are $3 \times 3$ tensors with normal components which allow surface relaxation due to underco-ordinated atoms lying at material surfaces. The normal components arise because the atoms that constitute the surface unit cells lack proper atomic co-ordination in the direction normal to the surface; therefore, the atomistic forces $U^{\prime}\left(r^{(j)}\right)$ that are normalized to stresses in (31) are also out of balance in the normal direction. Thus, surface relaxation is necessary in the normal direction to regain an equilibrated state.

In contrast, traditional surface elastic approaches [22,24] utilize a $2 \times 2$ stress tensor which depends only on the tangential components of the deformation, and thus cannot explain surface relaxation effects. Solving (30) requires an iterative process to solve the non-linear system of equations. The purpose of the iterative procedure is to determine the unknown FE nodal displacements $\mathbf{u}_{I}$ that minimize the energy functional $\Pi(\mathbf{u})$.

What has been accomplished in (30) is a systematic manner of obtaining continuum stress measures by calculating the system potential energy as a function of bulk and surface components. By correctly calculating the system energy, standard continuum mechanics relationships can be utilized to derive stress measures for usage in FE computations. The salient feature of Equation (30) is that as the surface area to volume ratio becomes larger, the surface area terms will dominate the energetic expression. Because the stresses required for the FE internal forces are calculated by differentiating the strain energy density, correctly accounting for the surface energy will naturally lead to the correct forces on surface nodes.

This situation is exactly that which occurs in nanoscale materials such as nanowires, quantum dots, nanoparticles and nanobeams; for these small scale structures, the finite surface energies will create surface stresses that can cause both surface relaxation into the bulk, as well as unique mechanical properties caused by the need to overcome the intrinsic surface stresses $[14,51,52]$. In contrast, if the volume of the material is significantly larger than the surface area, then the potential energy from the surface terms will be insignificant compared to the volumetric potential energy, and the material will feel no effect from the surface stresses. Thus, this model degenerates to a bulk Cauchy-Born model as the length scale of the material increases.

\subsection{Implementation in $1 D$}

In this section, we briefly illustrate the finite element implementation within a simple, onedimensional framework assuming second nearest neighbour atomic interactions. In particular, we detail the construction of the surface clusters and describe the energy and stress calculations.

Implementing the bulk portion of the Cauchy-Born model into an FE program is done exactly as has been done previously [27,44]. At each FE integration point $X_{G}$ as in Figure 3, a small atomic cluster surrounding the integration point is created. In particular, because an atom in a 1D atomic chain has four neighbours if interacting with its second nearest neighbours, each integration point $X_{G}$ in Figure 3 is constructed with four atomic neighbours located at positions $X_{\alpha i}, i=1 \rightarrow 4$. The initial atomic spacing $R_{0}$ is that which minimizes the energy of the atomic chain. The bond length between the integration point $X_{G}$ and each cluster atom located at $X_{\alpha i}$ is first deformed using the stretch tensor $C$, such that the strain energy at the integration point can be found. The derivatives of the energy density are then taken as in (30) to calculate the integration point stress. 


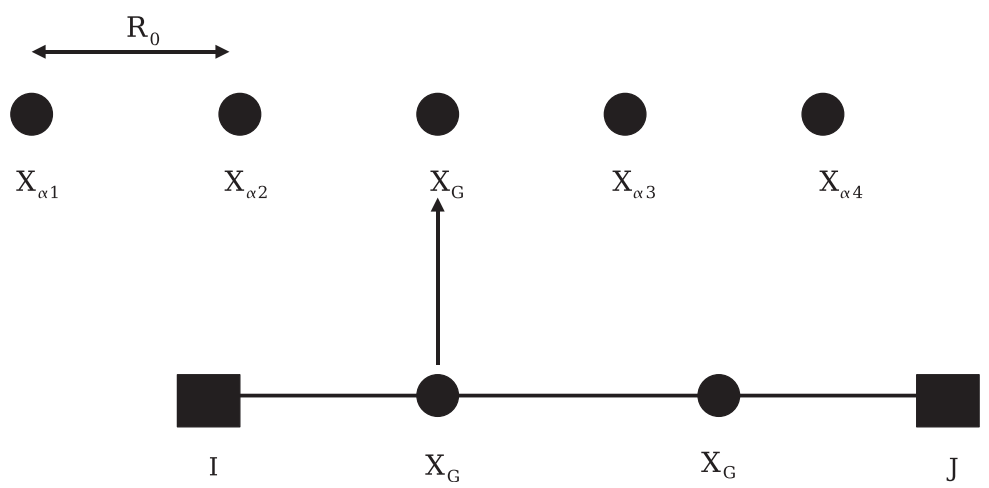

Figure 3. Initial positions of atomic neighbours $X_{\alpha}$ for finite element integration point $X_{G}$.

The only departure from standard FE techniques arises from the summation of the atomistic energies in (13), where it is noted that the summation over the bulk atoms goes from $\alpha=3 \rightarrow n-2$. In constructing the equivalent integral form to evaluate the energy, care must be taken to only integrate over the part of the domain which corresponds to the bulk. Thus, for elements which have at least one face that lies on a free surface, the volume integral term in (30) over $\Omega_{0}^{\text {bulk }}$ is not integrated over the entire element. Instead, it is integrated only over that portion of the element in which atomic behaviour is considered to be bulk. The remainder of the energy is accounted for by the area integrals in (30) over $\Gamma_{0}$, which are integrated over the non-bulk layers of atoms lying near the free surfaces.

Implementation of the surface clusters is done in a similar manner. In $1 \mathrm{D}$, evaluating the surface integrals in (30) is done pointwise, akin to enforcing an essential boundary condition. This is done by setting up surface clusters at two discrete points in the boundary finite elements. By utilizing surface clusters that accurately represent the degree of co-ordination of atoms at or near a surface, non-bulk effects are captured and directly integrated into the numerical calculation. The surface clusters represent the non-bulk energy terms in (17) and (18); the energies calculated from these clusters are used to evaluate the surface area integrals in (21) leading to the surface stresses in (31).

The first cluster is positioned at the boundary node itself, or directly on the surface of the body with location denoted by $X_{S}$ in Figure 4; because an atom lying on the surface of a 1D atomic chain would only have two neighbours if interacting with second nearest neighbours, the surface cluster point $X_{S}$ is also constructed with two neighbours. The spacing between cluster atoms is set up as in the bulk, with an atomic spacing of $R_{0}$. This cluster is illustrated in Figure 4, and is utilized to calculate the terms $\gamma_{1}(C)$ or equivalently $\gamma_{n}(C)$ in (17).

The second cluster is set up one atomic position $R_{0}$ inside the boundary node with location $X_{S}$ as illustrated in Figure 5. An atom one layer into the bulk in a $1 \mathrm{D}$ atomic chain interacting with second nearest neighbours would have three neighbours; therefore, this second cluster point is constructed with three neighbours, as illustrated in Figure 5. The spacing between cluster atoms is again $R_{0}$, and the cluster is utilized to calculate the terms $\gamma_{2}(C)$ or equivalently $\gamma_{n-1}(C)$ in (18). In $1 \mathrm{D}$, the stress for these clusters is equivalent to the force as the normalizing areas in (17) and (18) becomes unity. For both clusters, the procedure for evaluating the nodal 


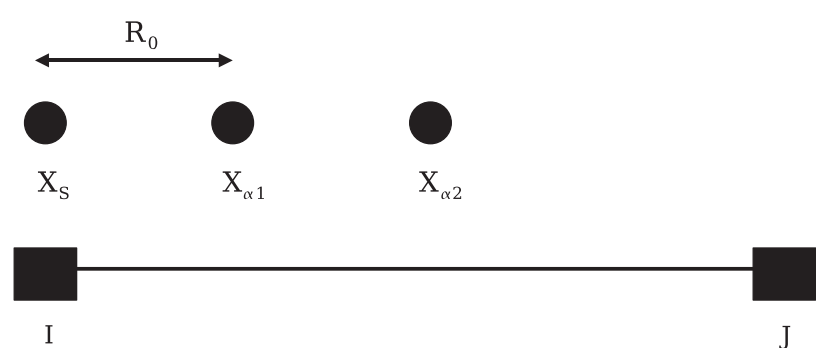

Figure 4. Initial positions of atomic neighbours $X_{\alpha}$ for surface cluster point $X_{S}$ that lies exactly on the free surface.

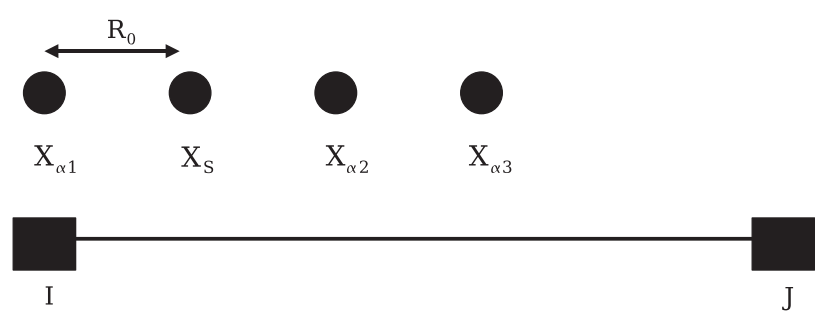

Figure 5. Initial positions of atomic neighbours $X_{\alpha}$ for surface cluster point $X_{S}$ that lies one atomic layer into the bulk.

forces on the boundary finite elements due to the surface clusters can be summarized as:

- Deform the initial bond lengths $R_{0}$ for each cluster point $X_{S}$ using the stretch tensor $C$ by following equation (8), i.e. $r=C^{1 / 2} R_{0}$.

- Evaluate the surface stress $\tilde{S}(C)$ at cluster point $X_{S}$ according to (31)

$$
\tilde{S}\left(X_{S}\right)=2 \frac{\mathrm{d} \gamma_{\Gamma_{0}}(C)}{\mathrm{d} C}=\frac{1}{\Gamma_{0}^{a}} \sum_{i=1}^{n_{s b}}\left(U^{\prime}\left(r^{(i)}\right) \frac{\mathrm{d} r^{(i)}}{\mathrm{d} C}\right)
$$

where $n_{s b}=2$ or 3 depending on whether the cluster is at the surface or one lattice parameter into the bulk and $\Gamma_{0}^{a}$ is unity in $1 \mathrm{D}$.

- Interpolate $\tilde{S}\left(X_{S}\right)$ to the finite element boundary nodes as

$$
\left(\begin{array}{c}
f_{I} \\
f_{J}
\end{array}\right)=\int_{\Gamma_{0}^{A}} \frac{\mathrm{d} N}{\mathrm{~d} X} \tilde{S} F^{\mathrm{T}} \mathrm{d} \Gamma=\left(\begin{array}{c}
\frac{\mathrm{d} N_{I}\left(X_{G}\right)}{\mathrm{d} X_{I}} \\
\frac{\mathrm{d} N_{J}\left(X_{G}\right)}{\mathrm{d} X_{J}}
\end{array}\right) \tilde{S}\left(X_{S}\right) F^{\mathrm{T}}
$$

Just as in an actual atomistic calculation, the forces on each of the surface cluster points $X_{S}$ in Figures 4 and 5 are found by evaluating the gradient of the interatomic potential where the initial bond lengths $R_{0}$ are deformed according to the stretch tensor $C$. The resulting force is normalized to a stress value by dividing by the appropriate initial unit cell area. In higher 
dimensions, the area normalization creates a stress-like quantity which is then plugged directly into the variational Equation (30).

\section{1D NUMERICAL EXAMPLE-SURFACE RELAXATION OF 1D BAR}

In the following 1D numerical example, 31 atoms interacting via a Lennard-Jones (LJ) 6-12 potential were modelled with second nearest neighbour interactions, with potential parameters $\sigma=\varepsilon=1$. The atoms were placed initially with interatomic distances $R_{0}=(4097 / 2080)^{1 / 6} \sigma$; this spacing was chosen as it represents the minimum energy configuration for a $1 \mathrm{D}$ lattice with periodic boundary conditions such that surface effects do not apply. In the general case in which atoms near the surfaces are not fully co-ordinated, this configuration leads to zero forces on every atom in the chain except for the second atom in from each boundary; this non-zero force then causes surface relaxation. An equivalent continuum FE model using the surface Cauchy-Born method developed earlier in this paper was used with a nodal spacing to interatomic distance ratio of $h / R_{0}=6$.

We verify the method in $1 \mathrm{D}$ by fixing the left end of the atomic chain, then allowing the remainder of the atoms in the lattice to relax to energy minimizing positions in a molecular statics (MS) calculation. Both the MS and FEM simulations were solved using a conjugate gradient method to minimize the energy. For this configuration, the right end of the atomic lattice expands by a normalized value of 0.0025 ; this value is exactly captured by the FE simulation.

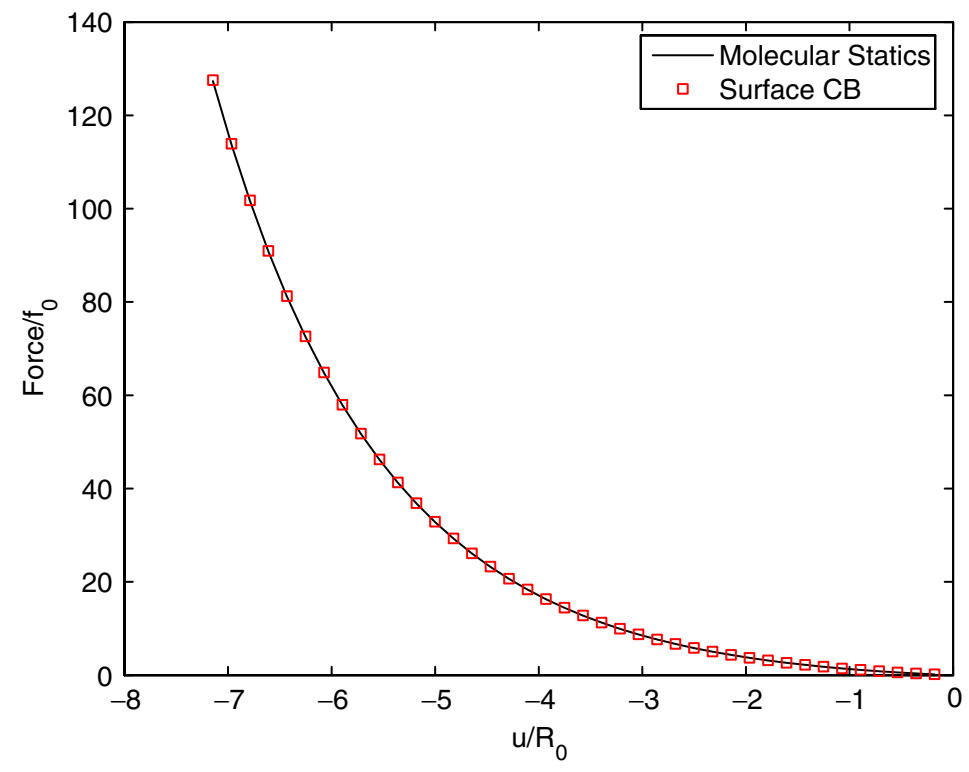

Figure 6. Load versus displacement for full molecular statics and surface Cauchy-Born model for compression of 1D atomic chain after initial relaxation. 
After relaxing the chain, the chain was loaded in compression by compressing the right end of the chain by a normalized value of 0.18 , equilibrating the chain, and repeating for successive load steps. The load versus displacement curve for the full MS and the FEM solution is shown in Figure 6, where the displacement has been normalized by the initial interatomic distance $R_{0}$, and the force has been normalized by the peak force of a nearest neighbour LJ potential. As can be seen, the surface Cauchy-Born solution accurately captures the non-linear force versus displacement curve.

While the 1D numerical example shown is overly simplistic as it does not account for surface energy interplay in multiple dimensions, it demonstrates that the method can exactly capture surface effects in an atomic chain by correctly calculating the underlying energetics. In particular, because there is no tangential deformation in $1 \mathrm{D}$, this example illustrates the ability of the proposed approach to capture the normal surface stresses that are required to drive surface stress-driven relaxation. We will proceed on in the next section to more complex and revealing $3 \mathrm{D}$ examples.

\section{3D NUMERICAL EXAMPLE-SURFACE RELAXATION OF 3D CUBE}

\subsection{Linear hexahedral elements}

We consider next the free surface relaxation of an FCC crystal that was modelled using both the proposed surface Cauchy-Born method and a fully atomistic MS calculation. The fully atomistic system had a cubic geometry with $21 \times 21 \times 21$ unit cells in the $x, y$ and $z$-directions, respectively, corresponding to 34461 atoms. The crystallographic orientation was $\left\langle\begin{array}{lll}1 & 0 & 0\end{array}\right)$ with $\left\{\begin{array}{lll}1 & 0 & 0\end{array}\right\}$ side surfaces. The same system was discretized with a regular mesh of 1728 8-node hexahedral elements with 2197 nodes, corresponding to a finite element mesh spacing to atomic spacing ratio of $h / R_{0}=2.36$. The atomic interactions were modelled using a LJ 6-12 potential with potential parameters $\sigma=\varepsilon=1$ with atomic interactions truncated after the third shell of nearest neighbours. The volume integral in (30) was integrated using $2 \times 2 \times 2$ Gaussian quadrature, while the surface integrals were evaluated using $2 \times 2$ Gaussian quadrature.

Denoting the length of the cube as $L$ with 0 being the midpoint, the atom at $(-L / 2$, $-L / 2,-L / 2)$ was fixed in all directions, the atom at $(L / 2,-L / 2,-L / 2)$ was fixed in the $y$ and $z$-directions, and the atom at $(-L / 2, L / 2,-L / 2)$ was fixed in the $z$-direction. These six essential boundary conditions ensure the removal of rigid body modes when relaxing the system. All other atoms and surfaces were without constraint. The FEM nodes corresponding to those atomic positions were fixed in the same manner. The MS energy was minimized again using a preconditioned conjugate gradient solver, while the FEM solution was obtained using a non-linear Newton solver.

A comparison of the MS displacement solution and the surface Cauchy-Born solution is shown in Figures 7 and 8. The figures illustrate that the proposed surface Cauchy-Born method qualitatively captures the correct displacement tendencies exhibited by the actual MS simulation. For example, the $-x$ face of the cube is found to expand in Figure 7 while the $+x$ face of the cube is also found to expand as seen in Figure 8 by the MS calculation. In both cases, the surface Cauchy-Born method captures the correct expansion tendencies. In addition, the surface Cauchy-Born correctly captures the gradients of the displacements near the corners of 

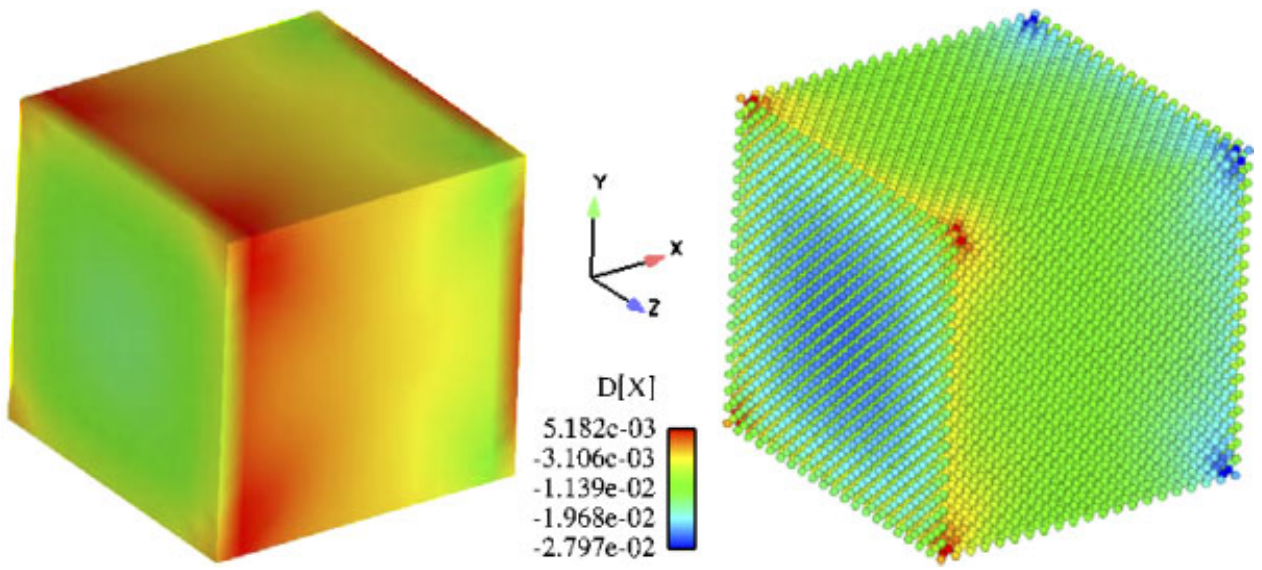

Figure 7. $x$-displacements as calculated by (left) surface Cauchy-Born method with linear elements and (right) molecular statics simulation.
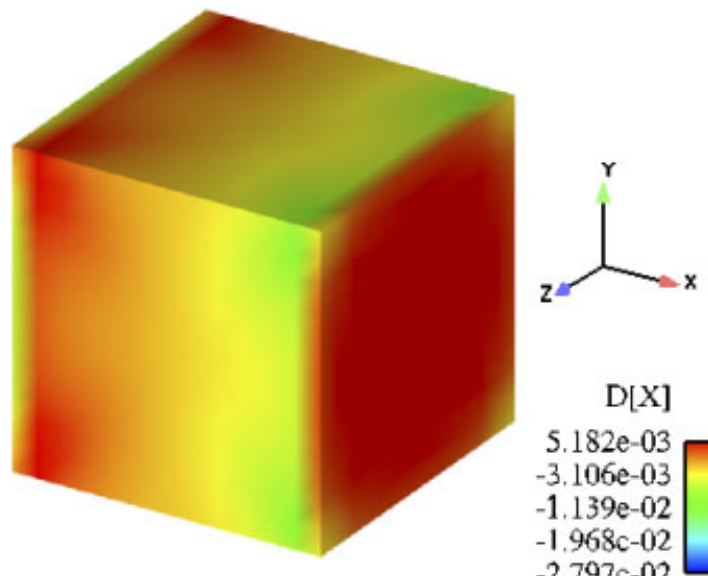

\section{$\mathrm{D}[\mathrm{X}]$}

$5.182 \mathrm{e}-03$

$-3.106 \mathrm{e}-03$

$-1.139 \mathrm{e}-02$

$-1.968 \mathrm{c}-02$

$-2,797 \mathrm{c}-02$

Figure 8. $x$-displacements as calculated by (left) surface Cauchy-Born method with linear elements and (right) molecular statics simulation.

the cube, where the atoms tend to contract inwards instead of expanding outwards as at the $-x$ and $+x$ free surfaces.

A comparison between the surface Cauchy-Born stresses and MS stresses as calculated on an atom-by-atom basis using the virial theorem is shown in Figure 9. Ignoring the kinetic portion of the virial as we are considering only quasistatic solutions, the virial theorem can be 

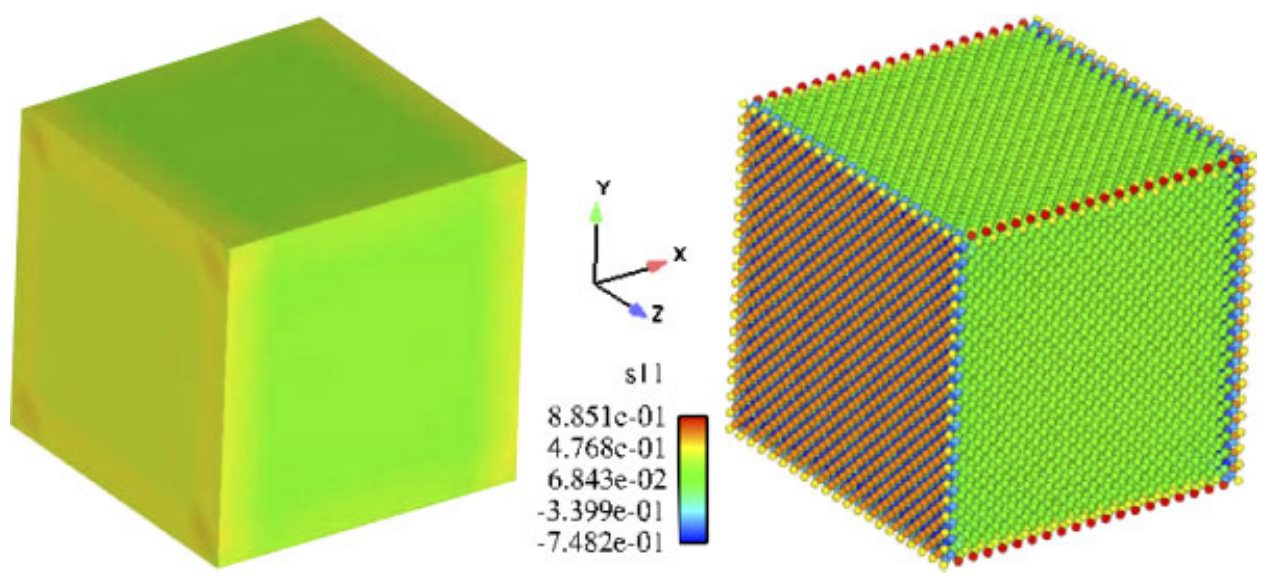

Figure 9. $\sigma_{x x}$ as calculated by (left) surface Cauchy-Born method with linear elements and (right) molecular statics simulation using the virial stress approximation.

written as [53]

$$
\sigma_{i j}^{\gamma}=\frac{1}{\Omega_{0}^{\gamma}}\left(\frac{1}{2} \sum_{\alpha=1}^{N} \sum_{\beta \neq \alpha}^{N} U^{\prime}\left(r^{\alpha \beta}\right) \frac{\Delta x_{i}^{\alpha \beta} \Delta x_{j}^{\alpha \beta}}{r^{\alpha \beta}}\right)
$$

where $\Omega_{0}^{\gamma}$ is the reference volume for each atom $\gamma, N$ is the total number of atoms, $r^{\alpha \beta}$ is the distance between two atoms $\alpha$ and $\beta, \Delta x_{j}^{\alpha \beta}=x_{j}^{\alpha}-x_{j}^{\beta}, U$ is the potential energy function and $r^{\alpha \beta}=\left\|\Delta x_{j}^{\alpha \beta}\right\|$.

As can be seen, the surface Cauchy-Born model using linear hexahedral elements is not able to capture the stress gradients near the free surface. While the stresses in the atomistic simulation show a checkerboarding pattern where the stress on the surface atom is positive followed by a negative stress on the next layer of atoms, the surface Cauchy-Born model interprets this stress in an average sense. Due to known inaccuracies with the virial stress at free surfaces [53], we are more interested in a qualitative comparison of stresses at the free surfaces, and the ability of the surface Cauchy-Born method to capture gradients of stress and energy at or near the free surfaces. This issue will be discussed further in the next section with regards to comparing surface Cauchy-Born and MS values of hydrostatic pressure.

A direct comparison of surface Cauchy-Born values of normal displacements and stresses at the centre of all free surfaces and the MS values are shown in Table I. As is expected, the surface Cauchy-Born qualitatively captures all the displacements and stresses as compared to the MS calculation; an exact matching of values is not expected due to the reduction in the number of degrees of freedom in the finite element model as compared to the fully atomistic representation. 
Table I. Comparison of stresses and displacements between surface Cauchy-Born method and molecular statics calculation. Displacements and stressed normalized by molecular statics values.

\begin{tabular}{lccccccc}
\hline Method & $u_{x}(-x)$ & $u_{x}(+x)$ & $u_{y}(-y)$ & $u_{y}(+y)$ & $u_{z}(-z)$ & $u_{z}(+z)$ & $\sigma_{11}(+x)$ \\
\hline Atomistics & -1.0 & 1.0 & -1.0 & 1.0 & -1.0 & 1.0 & 1.0 \\
Linear hex & -0.60 & 6.47 & -0.60 & 6.47 & -0.60 & 6.47 & 0.62 \\
Quad hex & -0.76 & 0.53 & -0.76 & 0.53 & -0.76 & 0.53 & 1.26 \\
\hline
\end{tabular}
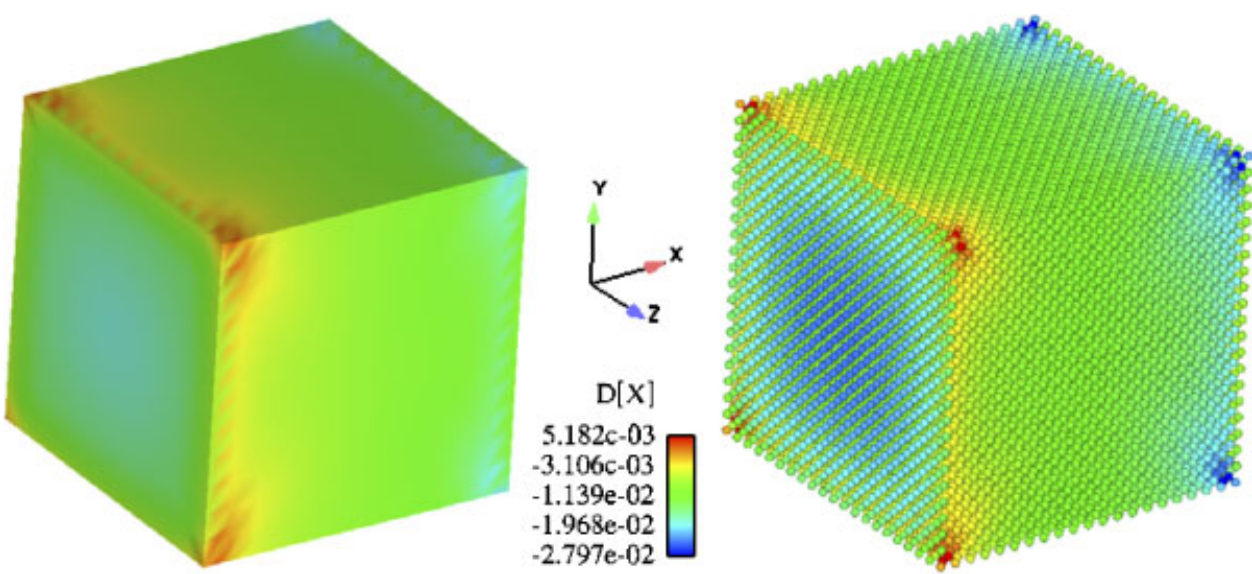

Figure 10. $x$-displacements as calculated by (left) surface Cauchy-Born method with quadratic elements and (right) molecular statics simulation.

\subsection{Quadratic hexahedral elements}

To examine the ability of the method to capture the high gradients of energy occurring near the free surfaces, quadratic 20-node hexahedral elements were employed to determine the utility of higher order elements in resolving the gradients. For the quadratic elements, the volume integral in (30) was integrated using $3 \times 3 \times 3$ Gaussian quadrature, while the surface integrals were evaluated using $3 \times 3$ Gaussian quadrature. Figures 10-12 show $x$-displacements and $\sigma_{x x}$ for a regular mesh of 20-node quadratic hexahedral elements corresponding to 1728 elements and 8281 nodes, leading to a finite element mesh spacing to atomic spacing ratio of $h / R_{0}=2.36$. As can be seen, the displacements and displacement gradients are captured much more accurately using the 20-node hexahedral elements. Moreover, as shown in Table I, the values for the $x$-displacements on the $+x$ and $-x$ faces are also more accurate than for the equivalent mesh of linear elements.

We compare the hydrostatic pressure along a line in the centre of the cube between the $-x$ and $+x$ faces for MS and both linear and quadratic elements. As can be seen in Figure 13, the proposed surface model correctly captures the bulk pressure away from the surface for both linear and quadratic elements, matching the molecular statics calculations. More importantly, the stress state in the interior of the cube is seen to be compressive, which is in agreement with recent atomistic simulations [54] showing that equilibration after surface-stress-driven relaxation occurs when the tensile surface stresses are balanced by a compressive stress state in the interior 

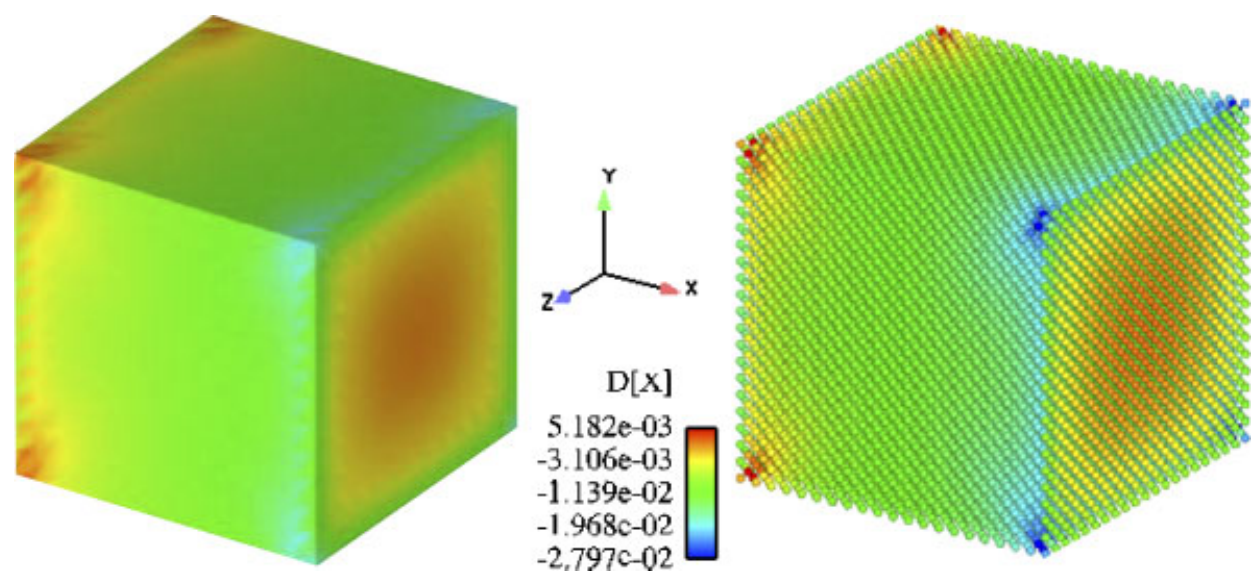

Figure 11. $x$-displacements as calculated by (left) surface Cauchy-Born method with quadratic elements and (right) molecular statics simulation.
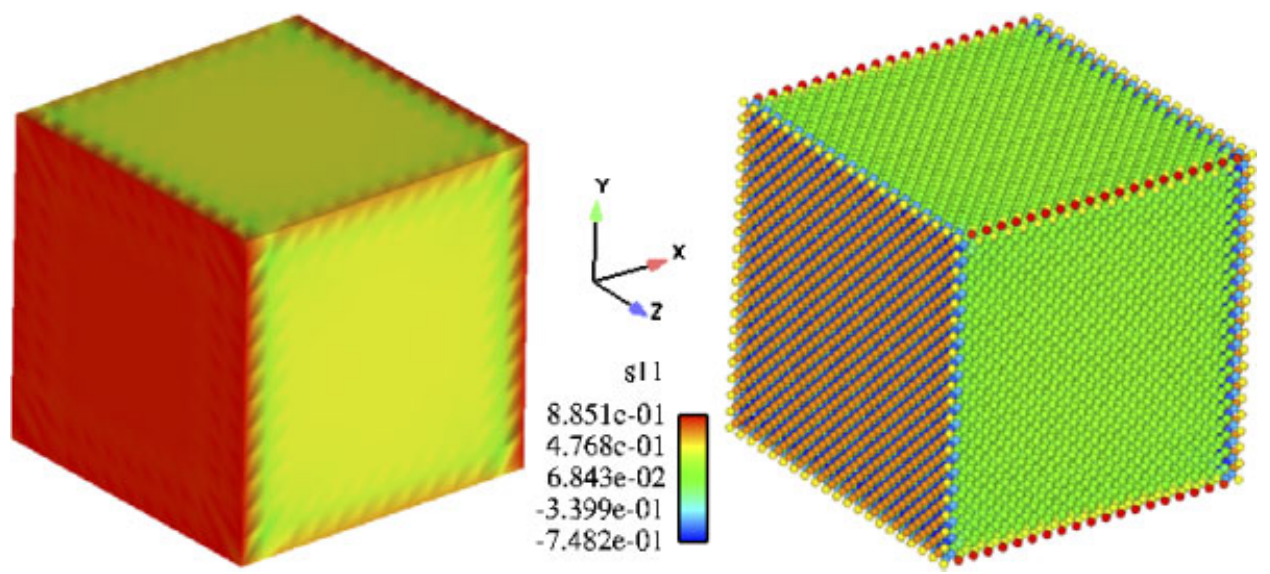

Figure 12. $\sigma_{x x}$ as calculated by (left) surface Cauchy-Born method with quadratic elements and (right) molecular statics simulation using the virial stress approximation.

of the material. However, with the FE discretization discussed above, the surface model using linear elements is unable to capture the high gradient in pressure at the surface; note that the physical space in Figure 13 between $x=-14.13$ and -9.34 is modelled using two elements, which explains the linear fit to the pressure between those two points.

In analysing the pressure as captured using the quadratic elements in Figure 13, it is evident that the high gradients near the free surfaces can be captured using higher order elements. The kinking of the pressure for the quadratic elements near the free surfaces can be attributed to the inability of the projection scheme utilized in the post-processing to capture the gradients in the pressure near the surface. The comparison to the MS pressure is not exact, but follows 


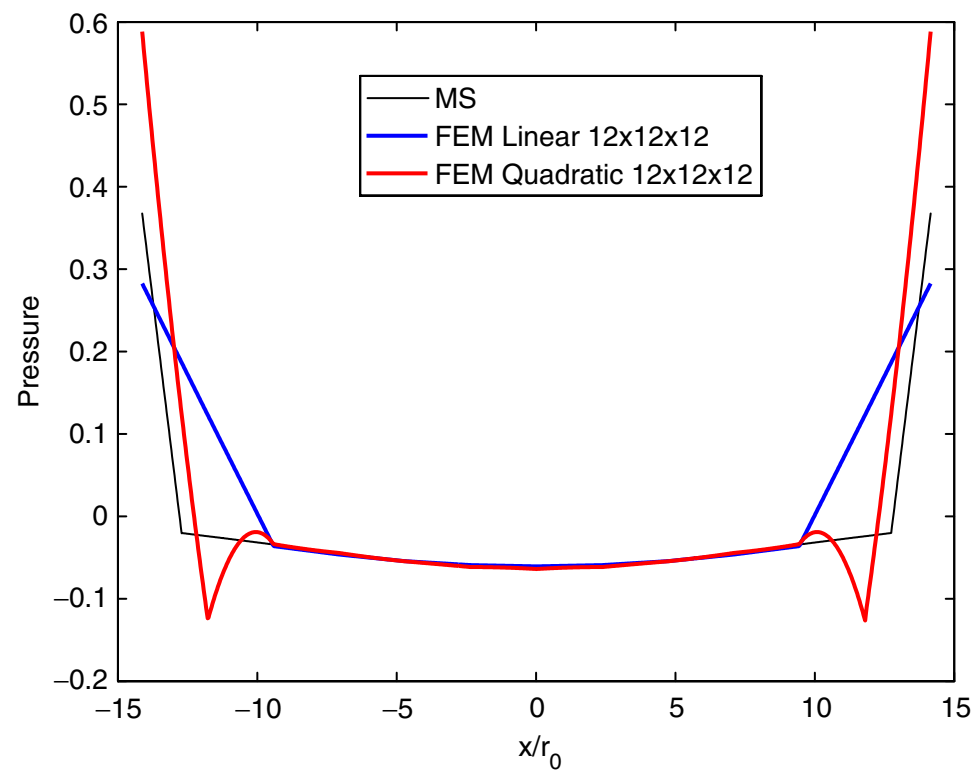

Figure 13. Hydrostatic pressure through the centre of the cube between the $-x$ and $+x$ faces for both proposed surface Cauchy-Born method with linear and quadratic elements and full molecular statics calculation.

the same qualitative behaviour. Due to known issues with the virial stress at free surfaces [53], what is important to note here is that quadratic elements can capture high gradients, both in pressure and in energy, near atomistic free surfaces, leading to the improved results seen in this section. On the other hand, the fact that the surface model compares well with the virial stresses in the bulk is also important, as the virial stress is known to give accurate results in bulk, non-defective lattices.

A final point to conclude the discussion on numerical results concerns the comparison of the proposed surface Cauchy-Born method to the standard Cauchy-Born approach. The standard Cauchy-Born approach, because of its treatment of all points as bulk, would predict zero surface relaxation for any problem and geometry. However, as we have shown and is wellknown, free surface effects clearly cause varying degrees of surface relaxation, which then impact the mechanical properties of nanoscale materials. Unlike the bulk model, the proposed surface Cauchy-Born method is able to capture these effects as predicted by molecular statics calculations.

\section{CONCLUSIONS}

We have developed a surface Cauchy-Born model to study the mechanics of nanoscale materials in which surface effects play a critical role in defining the mechanical properties. The approach is based upon decomposing the atomistic potential energy into bulk and surface components; by taking this direction, a simple variational equation is derived in which bulk and surface 
stresses constitute distinct terms in the variational equation. Furthermore, this decomposition lends itself to a physically intuitive idea of the approach; as the size of a continuum body increases, the volumetric portion of the energy will dominate the overall energetics of the system, and the surface effects will become negligible. On the other hand, when the length scale of the system approaches the nanometer scale, the surface energy terms will become crucial, and will contribute heavily to the total system energy.

While many elegant mathematical theories and representations of surface stresses on continuum bodies have been derived [18-26], the fact that the variational equation derived in this work can be discretized and solved using standard non-linear finite element techniques gives this approach the important advantage of being applicable to generalized boundary value problems involving nanomaterials of arbitrary geometry. Furthermore, the effects of surface stresses are captured in the constitutive response without recourse to additional atomistic simulations to fit elastic constants. In addition, by utilizing surface clusters to capture the fact that forces and thus stresses normal to a free surface are not in equilibrium, the proposed approach can represent the normal stresses that are necessary to capture surface stress driven relaxation. The fact that different surface orientations can be easily modelled by modifying the surface unit cells is critical, as it has been recently shown that side surface orientation can directly impact the deformation mode and mechanical properties of nanoscale materials [55].

Numerical examples in one- and three-dimensions were presented to verify and validate the proposed method. It was shown that the method performed nearly exactly in one-dimension, including surface relaxation and load-displacement behaviour in compression. For a threedimensional FCC cube, the proposed surface Cauchy-Born method qualitatively captured the mechanical response using a free surface relaxation example. In particular, the correct faces of the cube were observed to expand or contract as compared to a benchmark molecular statics simulation. Surface stresses and pressure were also found to compare qualitatively as well. Marked improvement in the numerical results was obtained by using quadratic elements, indicating that the energy variations near the surface can be captured effectively using higher order finite elements. In all cases, the surface Cauchy-Born method proposed here offers superior results than the standard Cauchy-Born formulation, which produces no surface relaxation.

The approach as currently formulated in this paper is certainly not applicable to all situations. For example, due to the homogeneous deformation assumption of the Cauchy-Born method, the method cannot capture surface reconstructions, defects emitted from the free surfaces, or surface stress driven phase transformations or reorientations. However, the method is still fully valid for non-linear elastic deformations, as is typical of crystal elastic formulations based on the Cauchy-Born rule.

In the future, we will pursue three research paths. First, we are formulating this method to utilize embedded atom (EAM) [46] potentials such that realistic metallic systems can be studied. This work would serve as a better indication of the method to model physically realistic systems, as it is well-known that anomalous surface relaxation and behaviour such as softening of the elastic constants [56] can occur using pair potentials. A second, and related task involves the application of this method to situations in which multiple unique surface facets (i.e. $\{100\}$, $\left\{\begin{array}{lll}1 & 1 & 0\end{array}\right\}$ and $\left.\left\{\begin{array}{lll}1 & 1 & 1\end{array}\right\}\right)$ are present at the same time. This situation is often seen in the analysis of nanoclusters [5,6], and will present an interesting test of the present approach. Finally, we will examine methods of improving the method itself by investigating internal degrees of freedom for the surface Cauchy-Born clusters $[47,48]$. 


\section{ACKNOWLEDGEMENTS}

HSP gratefully acknowledges startup funding from Vanderbilt University in support of this research. Sandia is a multiprogram laboratory operated by Sandia Corporation, a Lockheed Martin Company, for the United States Department of Energy's National Nuclear Security Administration under contract DE-AC04-94AL85000.

\section{REFERENCES}

1. Iijima S. Helical microtubules of graphitic carbon. Nature 1991; 354(6348):56-58.

2. Canham LT. Silicon quantum wire array fabricated by electrochemical and chemical dissolution of wafers. Applied Physics Letters 1990; 57(10):1046-1048.

3. Lieber CM. Nanoscale science and technology: building a big future from small things. MRS Bulletin 2003; 28(7):486-491.

4. Yang P. The chemistry and physics of semiconductor nanowires. MRS Bulletin 2005; 30(2):85-91.

5. Martin CR. Nanomaterials_a membrane-based synthetic approach. Science 1994; 266(5193):1961-1966.

6. Brust M, Walker M, Bethell D, Schriffrin D, Whyman R. Synthesis of thiol-derivatized gold nanoparticles in a 2-phase liquid-liquid system. Journal of the Chemical Society-Chemical Communications 1994; 7:801-802.

7. Arakawa Y, Sakaki H. Multidimensional quantum well laser and temperature-dependence of its threshold current. Applied Physics Letters 1982; 40(11):939-941.

8. Diao J, Gall K, Dunn ML. Surface-stress-induced phase transformation in metal nanowires. Nature Materials 2003; 2(10):656-660.

9. Gall K, Diao J, Dunn ML, Haftel M, Bernstein N, Mehl MJ. Tetragonal phase transformation in gold nanowires. Journal of Engineering Materials and Technology 2005; 127:417-422.

10. Park HS, Gall K, Zimmerman JA. Shape memory and pseudoelasticity in metal nanowires. Physical Review Letters 2005; 95:255504.

11. Park HS, Ji C. On the thermomechanical deformation of silver shape memory nanowires. Acta Materialia, accepted.

12. Liang W, Zhou M. Pseudoelasticity of single crystalline $\mathrm{Cu}$ nanowires through reversible lattice reorientations. Journal of Engineering Materials and Technology 2005; 127(4):423-433.

13. Park HS. Stress-induced martensitic phase transformation in intermetallic nickel aluminum nanowires. Nano Letters, accepted.

14. Gall K, Diao J, Dunn ML. The strength of gold nanowires. Nano Letters 2004; 4(12):2431-2436.

15. Park HS, Zimmerman JA. Modeling inelasticity and failure in gold nanowires. Physical Review B 2005; 72:054106.

16. Maranganti R, Sharma P. A review of strain field calculations in embedded quantum dots and wires. accepted.

17. Wong EW, Sheehan PE, Lieber CM. Nanobeam mechanics: elasticity, strength, and toughness of nanorods and nanotubes. Science 1997; 77:1971-1975.

18. Gurtin ME, Murdoch A. A continuum theory of elastic material surfaces. Archives of Rational Mechanics and Analysis 1975; 57:291-323.

19. Cammarata RC. Surface and interface stress effects in thin films. Progress in Surface Science 1994; 46(1):1-38.

20. Streitz FH, Cammarata RC, Sieradzki K. Surface-stress effects on elastic properties. I. Thin metal films. Physical Review B 1994; 49(15):10699-10706.

21. Miller RE, Shenoy VB. Size-dependent elastic properties of nanosized structural elements. Nanotechnology 2000; 11:139-147.

22. Shenoy VB. Atomistic calculations of elastic properties of metallic FCC crystal surfaces. Physical Review B 2005; 71:094104.

23. He LH, Lim CW, Wu BS. A continuum model for size-dependent deformation of elastic films of nano-scale thickness. International Journal of Solids and Structures 2004; 41:847-857.

24. Sharma P, Ganti S, Bhate N. Effect of surfaces on the size-dependent elastic state of nano-inhomogeneities. Applied Physics Letters 2003; 82(4):535-537.

25. Sun CT, Zhang H. Size-dependent elastic moduli of platelike nanomaterials. Journal of Applied Physics 2003; 92(2):1212-1218. 
26. Dingreville R, Qu J, Cherkaoui M. Surface free energy and its effect on the elastic behavior of nano-sized particles, wires and films. Journal of the Mechanics and Physics of Solids 2005; 53:1827-1854.

27. Tadmor E, Ortiz M, Phillips R. Quasicontinuum analysis of defects in solids. Philosophical Magazine A 1996; 73:1529-1563.

28. Shilkrot LE, Miller RE, Curtin WA. Multiscale plasticity modeling: coupled atomistics and discrete dislocation mechanics. Journal of the Mechanics and Physics of Solids 2004; 52:755-787.

29. Fish J, Chen W. Discrete-to-continuum bridging based on multigrid principles. Computer Methods in Applied Mechanics and Engineering 2004; 193:1693-1711.

30. Abraham FF, Broughton J, Bernstein N, Kaxiras E. Spanning the continuum to quantum length scales in a dynamic simulation of brittle fracture. Europhysics Letters 1998; 44:783-787.

31. Rudd RE, Broughton JQ. Coarse-grained molecular dynamics and the atomic limit of finite elements. Physical Review B 1998; 58:5893-5896.

32. E W, Huang ZY. A dynamic atomistic-continuum method for the simulation of crystalline materials. Journal of Computational Physics 2002; 182:234-261.

33. Wagner GJ, Liu WK. Coupling of atomistic and continuum simulations using a bridging scale decomposition. Journal of Computational Physics 2003; 190:249-274.

34. Park HS, Karpov EG, Liu WK, Klein PA. The bridging scale for two-dimensional atomistic/continuum coupling. Philosophical Magazine 2005; 85(1):79-113.

35. Park HS, Karpov EG, Klein PA, Liu WK. Three-dimensional bridging scale analysis of dynamic fracture. Journal of Computational Physics 2005; 207:588-609.

36. Park HS, Karpov EG, Liu WK. A temperature equation for coupled atomistic/continuum simulations. Computer Methods in Applied Mechanics and Engineering 2004; 193:1713-1732.

37. Xiao SP, Belytschko T. A bridging domain method for coupling continua with molecular dynamics. Computer Methods in Applied Mechanics and Engineering 2004; 193:1645-1669.

38. Liu WK, Karpov EG, Park HS. Nano Mechanics and Materials: Theory, Multiscale Methods and Applications. Wiley: New York, 2006.

39. Li X, E W. Multiscale modeling of the dynamics of solids at finite temperature. Journal of the Mechanics and Physics of Solids 2005; 53:1650-1685.

40. Liu WK, Karpov EG, Zhang S, Park HS. An introduction to computational nano mechanics and materials. Computer Methods in Applied Mechanics and Engineering 2004; 193:1529-1578.

41. Fleck NA, Muller GM, Ashby MF, Hutchinson JW. Strain gradient plasticity: theory and experiment. Acta Metallurgica et Materialia 1994; 42(2):475-487.

42. Fleck NA, Hutchinson JW. Strain gradient plasticity. Advances in Applied Mechanics 1997; 33:295-361.

43. Gao H, Huang Y, Nix WD, Hutchinson JW. Mechanism-based strain gradient plasticity-I. Theory. Journal of the Mechanics and Physics of Solids 1999; 47(6):1239-1263.

44. Klein PA. A virtual internal bond approach to modeling crack nucleation and growth. Ph.D. Thesis, Stanford University, 1999.

45. Arroyo M, Belytschko T. An atomistic-based finite deformation membrane for single layer crystalline films. Journal of the Mechanics and Physics of Solids 2002; 50:1941-1977.

46. Daw MS, Baskes MI. Embedded-atom method: derivation and application to impurities, surfaces, and other defects in metals. Physical Review B 1984; 29(12):6443-6453.

47. Tadmor EB, Smith GS, Bernstein N, Kaxiras E. Mixed finite element and atomistic formulation for complex crystals. Physical Review B 1999; 59(1):235-245.

48. Zhang P, Huang Y, Geubelle PH, Klein PA, Hwang KC. The elastic modulus of single-wall carbon nanotubes: a continuum analysis incorporating interatomic potentials. International Journal of Solids and Structures 2002; 39:3893-3906.

49. Belytschko T, Liu WK, Moran B. Nonlinear Finite Elements for Continua and Structures. Wiley: New York, 2002.

50. Hughes TJR. The Finite Element Method: Linear Static and Dynamic Finite Element Analysis. Prentice-Hall: Englewood Cliffs, NJ, 1987.

51. Diao J, Gall K, Dunn ML. Yield asymmetry in metal nanowires. Nano Letters 2004; 4(10):1863-1867.

52. Diao J, Gall K, Dunn ML. Atomistic simulation of the structure and elastic properties of gold nanowires. Journal of the Mechanics and Physics of Solids 2004; 52:1935-1962.

53. Zimmerman JA, Webb III EB, Hoyt JJ, Jones RE, Klein PA, Bammann DJ. Calculation of stress in atomistic simulation. Modelling and Simulation in Materials Science and Engineering 2004; 12:S319-S332. 
54. Diao J, Gall K, Dunn ML. Surface stress driven reorientation of gold nanowires. Physical Review B 2004; 70:075413.

55. Park HS, Gall K, Zimmerman JA. Deformation of FCC nanowires by twinning and slip. Journal of the Mechanics and Physics of Solids, accepted.

56. Zhou LG, Huang H. Are surfaces elastically softer or stiffer?. Applied Physics Letters 2004; 84(11): 1940-1942. 